\title{
SUPPORTING A PUBLIC HEALTH CARE VIRTUAL ORGANIZATION BY KNOWLEDGE TECHNOLOGIES
}

\author{
Mitja Jermol ${ }^{1}$ (mitja.jermol@ijs.si $)^{1}$, Nada Lavrac (nada.lavrac@ijs.si $^{1,2}{ }^{1}$ Tanja \\ Urbančic (tanja.urbancic@ijs.si) ${ }^{2,1}$,Tadeja Kopad ${ }^{3}$ \\ (1) Jožef Stefan Institute, Ljubljana, SLOVENIA, \\ (2) Nova Gorica Polytechnic, Nova Gorica, SLOVENIA \\ (3) Public Health Institute, Celje, SLOVENIA
}

\begin{abstract}
The organization of public health care in Slovenia is hierarchical: the national Institute of Public Health coordinates the activities of a network of regional Public Health Institutes whose functions are public health monitoring, organizing the public health activities and proposing and implementing actions for maintaining and improving public health. Public Health Institutes themselves coordinate particular activities of a regional network of hospitals, clinics, community health centers, individual health professionals and other health care resources. In this paper we investigate the system of public health of Slovenia, viewing it as a virtual organization breeding environment, propose a selection of knowledge technologies that can be used to support the management of public health, and discuss the advantages gained by treating the system of public health institutions as a virtual organization breeding environment.
\end{abstract}

\section{INTRODUCTION}

Effective medical prevention and good access to health care resources are important factors that affect citizens' welfare and quality of life. As such, these are important factors in strategic planning at the national level, as well as in planning at the regional and local community level. Large quantities of data collected by medical institutions and governmental public health institutions can serve as a valuable source of evidence that should be taken into account when deciding about priorities to be included into strategic plans, or when deciding about specific measures to solve a particular health care problem.

The current organization of public health care in Slovenia is hierarchical: the national Institute of Public Health (IPH) coordinates the activities of a network of regional Public Health Institutes (PHIs) whose functions are public health monitoring, organizing the public health activities and proposing and implementing actions for maintaining and improving public health. Public Health Institutes 
themselves coordinate a regional network of hospitals, clinics, individual health professionals and other health care resources involved in particular health care activities.

The network of regional Public Health Institutes (PHIs), coordinated by the national IPH, can be viewed as a virtual organization with relatively well-defined competencies and methods of collaboration. On the other hand, a regional network of various health care resources can be viewed as virtual organization breeding environment (Camarinha-Matos, 2003) (regional VBE) monitored and coordinated by the regional Public Health Institute. For a particular request (i.e., a 'business opportunity' in the terminology of virtual organizations) either from the national IPH or from local institutions (industry, local government,...) the local PHI organizes a virtual organization from the regional VBE to perform the requested task. This view can be extended to the national level: the regional VBEs forming the national virtual organization breeding environment (national VBE), coordinated either by the national IPH or by a regional PHI.

To be able to form an appropriate virtual organization, the IPH and PHIs need to know the capabilities and characteristics of all the health resources (institutions and individuals) of the national/regional VBE. Once a particular virtual organization is formed, the coordinating national IPH or the regional PHI needs to be capable of managing the virtual organization in order to adequately solve the task at hand. This can be done effectively by appropriate knowledge management (Smith, 2000).

Knowledge management can be supported by the use of knowledge technologies, in particular by data mining (Mladenić, 2003) and decision support (Mladenić, 2003), which are in the focus of the work described in this paper. Data mining and decision support have a large potential for knowledge management in virtual organizations, and have already proved to be successful in numerous applications. Data mining is typically applied to knowledge discovery in large and complex databases and has been extensively used in industrial and business problem solving, while its use in health care is still rare. In such a knowledge intensive domain, neither data gathering nor data analysis can be successful without using knowledge about both the problem domain and the data analysis process, which indicates the usefulness of integrating data mining with decision support techniques to promote the construction of effective decision models supporting decision making and planning in public health care.

This paper investigates the system of public health of Slovenia, viewing it as a virtual organization breeding environment and proposing a selection of knowledge technologies that can be used to support the management of public health. Section 2 provides a framework for knowledge management in virtual organizations. Section 3 describes the organization of public health care in Slovenia, viewing the network of Slovenian health care resources as a virtual organization breeding environment and points out the advantages of taking such a view for improved knowledge management. Section 4 identities some problems that can be solved with the use of knowledge technologies and proposes the specific knowledge technologies, data mining and decision support, for solving particular problems identified by the Public Health Institute of the Celje region, chosen as the referential PHI. The paper concludes with some lessons learned and directions for further work. 


\section{KNOWLEDGE MANAGEMENT IN VIRTUAL ORGANIZATIONS}

The main strength of a virtual organization lies in the range of competencies the partners are able to offer jointly through collaboration. To fully exploit this advantage, problems of efficiently storing, updating, sharing, promoting and transferring knowledge need to be solved. These problems are solved by appropriate knowledge management which - in addition to the technological solutions - needs to address also organizational, economic, legislative, psychological and cultural issues (McKenzie, 2001). Knowledge management is recognized as the main paradigm for successful management of virtual organizations, aimed at supporting business intelligence (Biere, 2003) - a broad category of applications and technologies for gathering, storing, analyzing, and providing access to data to help organizations make better business decisions. Business intelligence applications include decision support (Mladenić, 2003), query and reporting, online analytical processing (OLAP), statistical analysis, forecasting, and data mining (Mladenić, 2003).

In virtual organizations, the strategic and management decision making processes typically differ from those in traditional organizations, where decisions are made at the top and implemented at the bottom. The model of managing a virtual organization can vary from a classical and ordered management system to selforganization. In any case, to manage a networked structure, well-defined guidelines are useful as a help to plan, control and finish a project in planned time.

A partner in a virtual enterprise can be viewed as an agent which can either be an individual, a group/team or an institution. An agent is a resource capable of performing a particular task in the project and/or the co-ordination between different processes for which it is responsible. The role of directing is performed by the agent acting as a project leader in the process of planning, leading and controlling a particular project in the virtual organization. For the sake of effective network management, it is very useful if the network intelligence (NQ) (Palmer, 1998) philosophy is adopted by the partners. Network intelligence states that an individual is a much stronger and valuable knowledge resource if it is a 'connected' member of the community. This holds also in the case of a virtual organization, where the added-value and the main strength of each partner is due to linking his expertise with supplementary and complementary knowledge of other partners. Moreover, also the most important strength of the whole virtual enterprise lies in the range of competencies the partners can offer together. The wider is the network of knowledge the more competitive it is as a whole.

For these purposes every partner has to have access to a knowledge resource repository, where the information about knowledge resources, process costs and resource availability in the network are stored. To be able to manage the network and perform successful knowledge management, an appropriate tool has to be selected. A suitable tool to support the above mentioned functions is a knowledge map, i.e., "a visual representation of a knowledge domain according to criteria that facilitate the location, comprehension or development of knowledge" (Eppler, 2003). The process used to gather the information needed for knowledge map construction is called knowledge mapping.

A knowledge map of partners' core competencies, additional expertise, tools and procedures in terms of explicit knowledge covers most of the knowledge 
management and business needs of a concrete business environment. According to (Burn, 2002), there are different types of knowledge maps: knowledge agent maps, knowledge asset maps, knowledge structure maps, knowledge development maps, and knowledge flow maps. A comprehensive knowledge map can be seen as a dynamic landscape of explicit knowledge (core competencies, legacy systems, additional expertise, forms, documents, presentations, papers), procedures (guidance, tips, checklists), tools and methods (web services, training, applications) available to the virtual organization. Some tacit knowledge (e.g., group dynamics, embodied knowledge and psychological) can be made explicit and added as well. A knowledge map has to be made accessible to all partners, in particular to the strategic decision makers.

When a new task or request (a 'business opportunity') arises, network partners use electronic means for discussing a new problem, trying to find out the best methods, techniques, tools and expert teams to develop a problem solution. This provides a suitable environment for the development of communities of practice (COPs) (Wenger, 1999) and virtual organization breeding environments (VBEs). On the one hand, COPs and VBEs are formed on the basis of agent's interests and form the grounds for the building of trust, collaboration, knowledge transfer and new knowledge discovery. On the other hand, the virtual organization itself can be developed into a community of practice or even into a virtual organization breeding environment.

\section{THE NETWORK OF SLOVENIAN HEALTH CARE RESOURCES VIEWED AS A VIRTUAL ORGANIZATION BREEDING ENVIRONMENT}

In Slovenia, the system of public health is organized at three levels: the strategic (national) level, the managerial (regional) level, and the operational (local) level.

- At the strategic level, the national Institute of Public Health (IPH) implements the national health care policy whose main concern is good public health and efficient national health care system which includes the appropriate distribution of health care resources across the regions of Slovenia.

- At the managerial level, the regional Public Health Institutes (PHI) propose and implement actions for maintaining and improving public health within the directives of the national IPH through public health monitoring and organizing public health activities.

- At the operational level, hospitals, clinics, individual health professionals and other health care resources offer the health services to the citizen; their activities need to be in line with the regional and the national public health policy.

Figure 1 illustrates the three level organization of public health care of Slovenia. The strategic level consists of a single institution (IPH), the managerial level consists of a network of regional Public Health Institutes (PHIs) while the institutions at the operational level form regional medical and health care networks. 


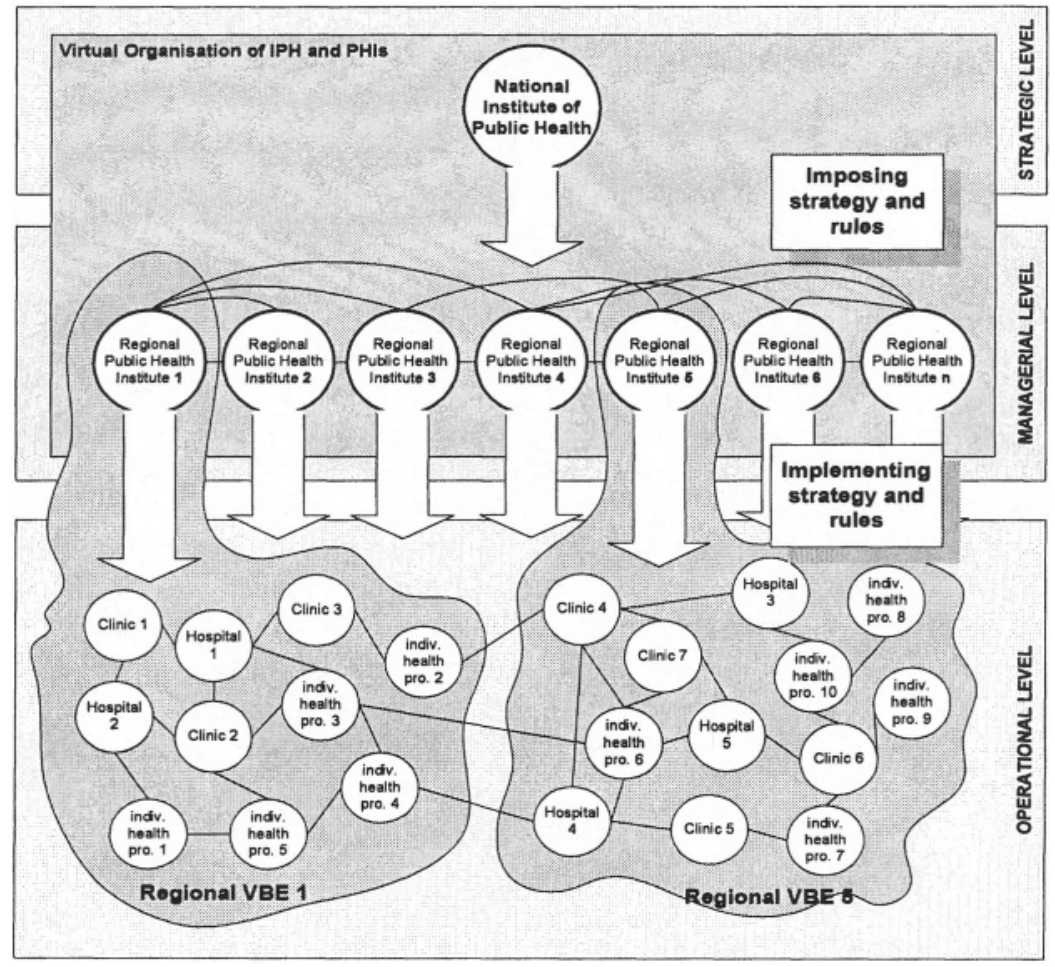

Figure 1 - The three level organization of public health care of Slovenia.

As discussed in the introduction of this paper, the network of regional Public Health Institutes (PHIs), coordinated by the national IPH, can be viewed as a virtual organization with relatively well-defined competencies and methods of collaboration. Other virtual organizations can be formed from the virtual organization breeding environment (VBE) at the operational level: local health institutions and professionals which act in accordance with the rules and procedures defined roughly by governmental policies and implemented by IPH at the national level and PHIs at the regional level.

By adopting the latter view, the entire national public health system of Slovenia can be seen as a network of different institutions and professionals (public health resources), which jointly function in accordance with the rules and procedures defined roughly by governmental policies and implemented by IPH and PHIs at the national and regional levels, respectively. Public health resources therefore form a loosely formalized association which, as a whole, can be viewed as a virtual organization breeding environment.

Every health care request triggers appropriate public health resources to form a firmer association, which results in a particular virtual organization formed for a given task. This is why public health resources at the regional operational level already function as a regional VBE. Let us illustrate the virtual organization life cycle for two different problem situations. 
- The first is a traditional one, where a patient comes to the general practitioner (GP) due to a health problem. The GP prescribes laboratory testing and based on the outcome directs the patient to a specialist who first prescribes medical treatment, but finally requests the patient to be treated in the local hospital. The actors in this process must act in line with the existing rules and procedures. If viewed from a virtual organization perspective, the rules prescribing the formation of a 'virtual organization' for this 'business opportunity' are predefined (partly by IPH and PHI) unless the patient decides to act differently than suggested by the health system procedures. The established virtual organization dissolves by the end of the treatment of the patient's health problem.

- A less standard situation occurs in preventive health care, in which a particular task can be triggered by local industry or a local community. Suppose that a group of people living in the same environment shows symptoms of the same health problem. In this case, this 'business opportunity' becomes the concern of a regional PHI, that needs to act as a 'net-broker' in the VBE consisting of resources at the operational level, resulting in the formation of an appropriate 'virtual organization' of health care resources for the given task.

The second example is a case in which a regional PHI is contacted in order to coordinate a specific activity. As the current national health reform is radically cutting the budget for PHIs, forcing them to offer their services to the market, such a request actually represents a business opportunity for the coordinating PHI. The virtual organization of PHIs is actually facing a rapid transformation process towards the inclusion into a national VBE formed of existing regional VBEs of public health resources. Their business opportunities come from local industries or local communities, as described in the second example above; in this case, a PHI acts as a net-broker. On the other hand, their business opportunities may be invoked by a request of the national Institute for Public Health (IPH) to several PHIs; in this case, the net-broker for this strategic level task is the national IPH itself.

The main goal of each regional PHI is to enable equable health development in the region by establishing and maintaining an effective regional public health system. This task depends on the proper organization and efficient management of regional health care resources in the health care VBE. To be successful in the market, the regional PHIs are thus facing new challenges, which are related to the management of VBEs. Hence, management and organizational concepts of VBEs and virtual organizations (VOs), originally developed for the production sector, need to be introduced also into the public health care sector.

\section{USING ADVANCED KNOWLEDGE TECHNOLOGIES TO SUPPORT THE PUBLIC HEALTH CARE VIRTUAL ORGANIZATION BREEDING ENVIRONMENT}

In Section 2 we have argued that the efficient management of VBEs and VOs critically depends on appropriate knowledge management, which is supported by knowledge technologies. The basis for an integrated knowledge management system are data and knowledge stored in databases and/or available through various communication technologies, the most important part of it being the database of 
knowledge contents (knowledge resources, knowledge processes) usually referred as the knowledge repository. The most successful tool used for the representation of the content in the knowledge repository is a knowledge map. In the knowledge map each knowledge resource (VBE member) is described by different sets of attributes, describing their knowledge and competences, availability, cost, time and quality to perform particular knowledge tasks.

As mentioned in Section 2, the processes used for gathering and updating the data and the knowledge repository are knowledge mapping processes. Automatic mapping processes are based on advanced knowledge technologies like data mining, text and web mining. Knowledge technologies used to represent contents in the knowledge map range from data mining, text and web mining to decision support tools.

A knowledge map can be a very useful tool to support the management of the VBE as well as VOs. In the case of a regional PHI, the management of a regional public health VBE depends on successful planning which should be based on an accurate analysis of the current situation. For this purpose, regional PHIs are already collecting some data from the regional VBE, which are used to perform some basic statistical analysis and personal judgment. But the most important information and knowledge remain hidden and therefore unused. This is why PHIs knowledge about a regional VBE and its members is fuzzy and inaccurate. This is one of the major problems to be solved if aiming at optimizing the organization of public health care. This problem is particularly emphasized in the case of unequal territorial distribution of public health resources, which results in the problem of unsuitable public health services provided to the citizens.

In the MediMap project for the regional PHI Celje we have tried to set up an appropriate model and the appropriate tools to support the regional health care VBE which could then serve as a model for other regional PHIs. We have focused on the development of knowledge technology tools, which may help solving the problem of VBE management (in terms of equal knowledge resource distribution, new knowledge resource acquisition, VBE members retention), VO formation and VO management (optimization, simulation, prediction, monitoring). These requirements, formulated by the PHI Celje, fall into three problem areas: (1) health care organization (the public health institute network, health care human resource distribution), (2) accessibility of health care services to the citizens, and (3) the health care providers work. They were made operational as five problem tasks:

- analysis of the public health providers network,

- analysis of public health human resources,

- analysis of public health providers workload,

- management and optimisation of the public health providers network, and

- simulations and prediction of the performance of the public health providers and human resource network.

In order to model the Celje regional VBE we wished to better understand all the Celje region resources and their connections. For that purpose data mining techniques have been applied to the data of ten community health centers. The dataset was formed of three databases:

- the health care providers database, 
- the out-patient health care statistics database (patients' visits to general practitioners and specialists, diseases, human resources and availability), and

- the medical status database.

To model the processes of a particular community health center (the patient flow), data describing the directing of patients to other community health centers or specialists were used. Our intention was twofold: on the one side we wanted to detect the similarities between community health centers and on the other to detect the atypical community health centers. Similarities between community health centers were analyzed according to four different categories: patient's age categories, patient's social categories, the organization of the community health center, and employment structure of the community health center.

For each category similarity groups were automatically constructed using four different clustering methods (detailed description of the clustering methods cannot be provided due to space constraints). Averages over four clustering methods per category were used to detect the similarities between the community health centres of the Celje region. The similarities are presented in Figure 2.

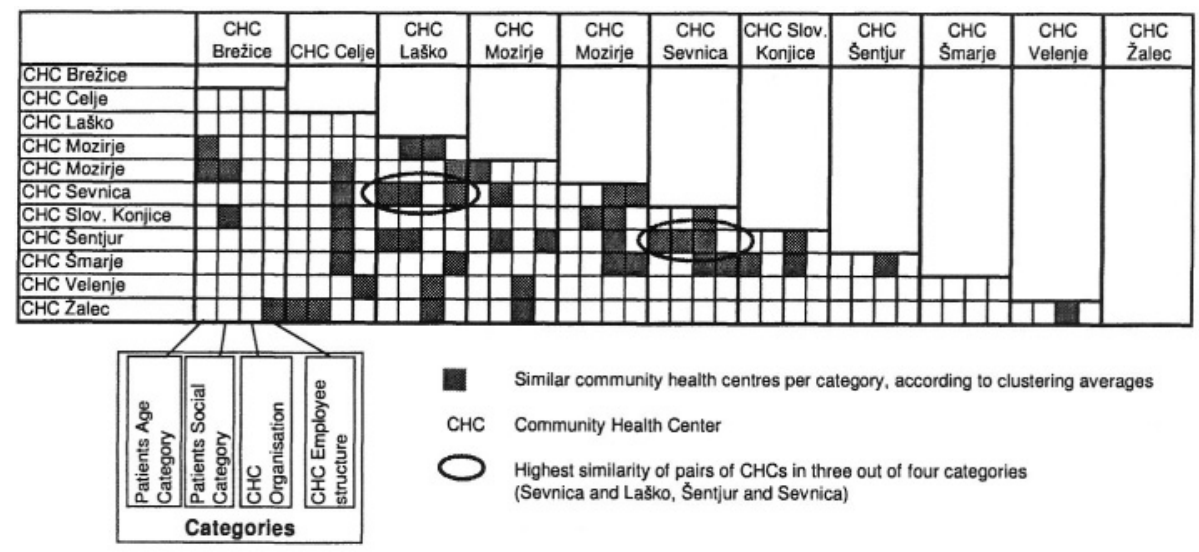

Figure 2- The similarity matrix of Celje CHCs.

Results were evaluated by domain experts from PHI Celje. In several cases the results confirmed already known similarities, while the experts could not find any reasonable explanations for new knowledge described in the similarity matrix, as the data describing was to coarse (aggregated).

A more detailed similarity analysis was therefore needed. In the analysis of the estimated number of patients that can be handled by a community health center (the center's capacity estimated by the number of employed staff), and the actual number of patients handled by the center, the outcome shown in Figure 3 was well appreciated by the experts. The figure presents some atypical centers (e.g., CHC Brežice and Žalec) with an insufficient number of staff compared to the number of actual patients. In general, the results from these experiments confirmed some similarities between community health centers and pointed out atypical community health centers, which was well accepted. A similar analysis as the one shown in Figure 3 was done for the regional distribution of drugstores which also showed 
atypical cases which need to be analyzed by the Public Health Institute Celje in further work.

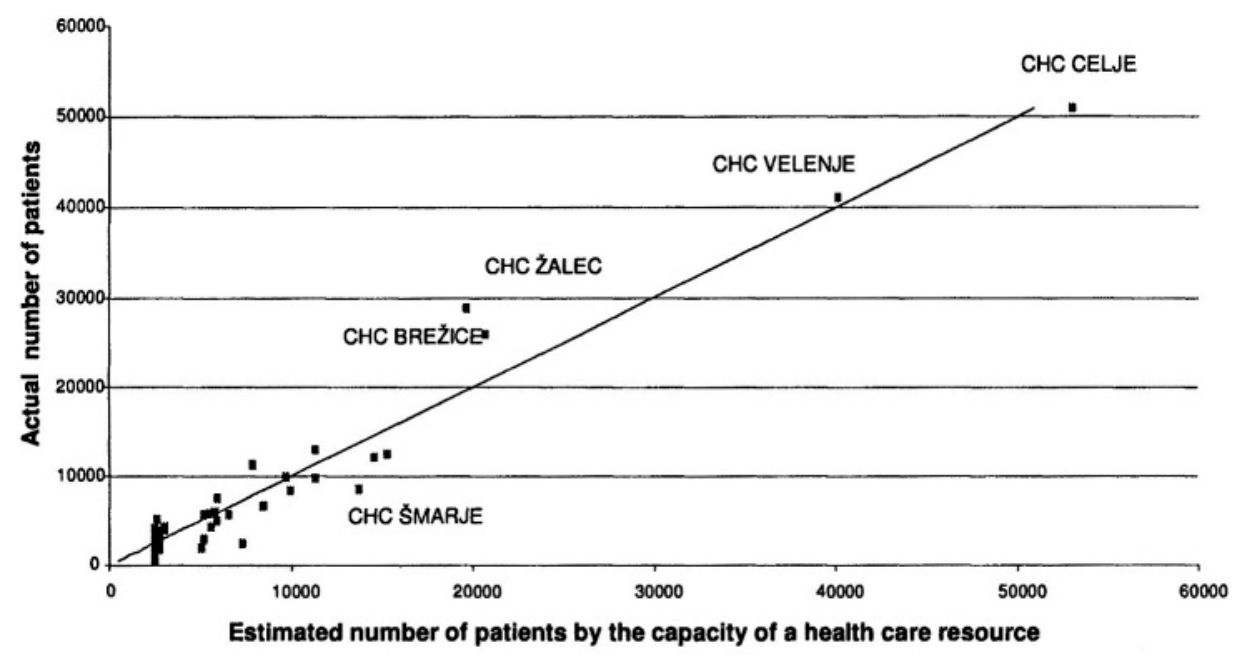

Figure 3- Detecting atypical Celje region health care resources (those deviating from the diagonal line).

Overall results indicate that additional data from the community health center will need to be gathered. This finding is valuable for the national IPH, which defines the data model and pre-describes rules and procedures for data gathering. This also fits our goal to build a knowledge repository and knowledge mapping procedures, which will support the management of the regional public health VBE. Another important aspect for the management of VBE is to automatically define similar community health centers and point out the extreme and atypical ones.

\section{CONCLUSION}

The organization of public health system in Slovenia can be viewed as a set of regional VBEs of public health resources which are managed by regional public health institutes (PHIs). Successful management of a virtual organisation breeding environment critically depends on up-to-date information about all VBE members and their mutual interactions. The most important information includes VBE member's core competences and knowledge resources. Processes of collecting, storing, maintaining and updating knowledge are used to gain better understanding of every VBE member. Sharing, promoting and transferring knowledge between VBE members facilitates and motivates the potential formation of virtual organizations. All mentioned processes can be supported by the appropriate knowledge map which enables decision makers to successfully manage the VBE and potential virtual organizations.

In the MediMap project we are developing methods and tools that will help regional PHIs and national IPH to perform their tasks more efficiently. Tools and 
methods were developed for the reference case of IPH Celje and tested on selected problems related to health care organization, accessibility of health care services to the citizens, and the health care providers work.

In the first part of the project some traditional statistical and data mining methods were used in order to get acquainted with the problem domain and data sources. In further work we will follow two objectives: (1) the development of a public health care knowledge map based on automatic knowledge gathering tools and (2) the development of decision support tools with automatic modeling of VBE members using data mining. We will focus on the development of decision support tools with the automatic modeling of VBE members using data mining.

The developed methodology and tools have proved to be appropriate to support VBE management. IPH Celje will directly benefit from these results which will in case of successful implementation, serve as a reference model for other regional IPHs. In addition, the national IPH is interested in the identification of missing data that should be included in the improved protocol of public health data gathering at the national level.

\section{Acknowledgements}

We gratefully acknowledge the Public Health Institute Celje and the Slovenian Ministry of Education, Science and Sport for funding the MediMap project, and to the MediMap team, in particular to Marko Debeljak, Marko Bohanec and Aleksander Pur who contributed to the results described in this paper.

\section{REFERENCES}

1. Biere M. Business Intelligence for the Enterprise, Prentice Hall PTR; 1st edition June 4, 2003

2. Burn MJ, Ash C. Managing Knowledge for Strategic Advantages in the Virtual Organisation. In Malhotra Y. (editor), Knowledge Management and Virtual Organizations, 144-161, Idea Publishing Group, USA, 2002.

3. Camarinha-Matos, L.M., Afsarmanesh H. Elements of a base VE infrastructure. J. Computers in Industry, Vol. 51, No. 2, 139-163, 2003

4. Eppler, MJ. Knowledge Management Terminology. Guide, St. Gallen; mcm institute, 1999, 07.2000. URL: <http://www.informationobjects.ch/NetAcademy/naservice/publications.nsf/all_pk/1617> [13.11.2003].

5. McKenzie J, van Winkelen C. Exploring E-collaboration Space. Henley Knowledge Management Forum, 2001.

6. Mladenic D, Lavrax N, Bohanec M, and Moyle S. (editors) Data Mining and Decision Support: Integration and Collaboration, Kluwer, 2003.

7. Palmer, J. The Human Organization. Journal of Knowledge Management, Vol. 1, Number 4, 294-307, June 1998.

8. Smith RG, Farquhar A. The Road Ahead for Knowledge Management: An AI Perspective. AI Magazine, Vol. 21, No. 4, 17-40, 2000.

9. Wenger E. Communities of Practice: Learning, Meaning, and Identity. Cambridge University Press, 1999. 